\title{
A generalized Frame Adaptive MPC for the low-level control of UAVs
}

\author{
Emil Fresk and George Nikolakopoulos
}

\begin{abstract}
The aim of this article is to establish an adaptive Model Predictive Control (MPC) scheme for the angular rate and thrust control of a multirotor Unmanned Aerial Vehicle (UAV). The proposed model adaptiveness comes from estimating the movement of the Center of Gravity (CoG) combined with the thrust constant of the motors, making the system robust to disturbances and fast to adapt to changing parameters, while also taking under consideration the control signal bounds in order to guarantee for no motor stalls, while flying. The linear requirements of the MPC are adhered to by transforming the estimation and control problem into a control signal squared domain, making the system linear. The efficacy of the proposed estimation and control scheme is presented in simulations where worst case scenarios have been considered.
\end{abstract}

\section{INTRODUCTION}

The field of UAVs has flooded the interest of researchers in the last decade, mainly due to the potential impact of aerial robotics in numerous real life applications, such as rural area monitoring [1], infrastructure inspection [2], mapping and general vision based aerial robotics [3].

Nowadays, there is a paradigm shift in the field of UAVs that is focusing on the field of physical interaction with the environment, such as the application scenarios of pick and place or load lifting applications. Characteristic examples that could be mentioned are in [4], where a helicopter combined with a gripper was developed for load transportation, in [5], where a quadrotor was used for building simple structures by combining it with a gripper mounted to its base, while also developing a novel construction algorithm, while in [6] an upward facing gripper was used to perform tasks at high altitudes and general manipulation of objects. Finally, in [7], payload transportation, using multiple quadrotors, was proposed with the aim to achieve a specific attitude and position of the payload using cables.

In all these applications, accurate control is an essential need to complete the mission with any kind of accuracy and repeatability with respect to the precision in the manipulation tasks and in the overall stability of the system. In the case of aerial inspection missions, the existing models (kinematic or dynamic) for UAVs and the corresponding control schemes [8], [9] are providing a very accurate and functional robotic system. However, in the cases where interaction with the environment is needed, e.g. manipulation on an object, there is an additional effect that should be taken under

This work has been partially funded by the European Unions Horizon 2020 Research and Innovation Programme under the Grant Agreement No.644128 AEROWORKS and the Grant Agreement No. 730302 SIMS.

The authors are with the Department of Computer Science, Electrical and Space Engineering at Luleå University of Technology, SE-97187, Luleå, Sweden.

Corresponding Author's E-mail: emil.fresk@1tu.se consideration during the modeling and the corresponding control scheme, which is the moving CoG.

In these cases, the time-varying nature of the $\mathrm{CoG}$ e.g. movement of a floating manipulator, or transportation of a load that swings, or pick and place maneuvers on objects, needs to be tracked and embedded in the corresponding modeling and control schemes, in order to guarantee the overall stability of the system and the desired performance. For the compensation of this time-varying alteration of $\mathrm{CoG}$ and the corresponding time-varying models, the classical approaches in the area of multirotors (mainly quadrotors) so far have been focusing on identifying parameters for static model systems as it can be identified in the following articles [10], [11] to name a few. The adaptation to changes in the $\mathrm{CoG}$ on quadrotors were investigated by [12], where a static gripper was used to move objects. In these cases, the simple geometry of the quadrotor, allowed the problem to be easily solved, while the major aim of this article was to improve this with a more general approach and to further validate it against time-varying $\mathrm{CoG}$ changes, including step changes, where [12] considered only step changes while transporting loads.

Recently, in [13] the authors have established a novel generalized representation of the $\mathrm{CoG}$ for the case of multirotor frames that was intuitive to use, as well as an online adaptive physical model for estimating the time-varying parameters of the system. Based on this framework, the major novelty of this article stems from the establishment of a time-varying MPC that it is able to handle the time-varying nature of the $\mathrm{CoG}$, while also adapting to the varying available thrust and mass of the system. The combination of the time-varying online estimation scheme with the corresponding proposed time-varying ability of the control scheme has the potential to create a solid theoretical foundation for the application of more advanced control and manipulation schemes as the challenges of varying $\mathrm{CoG}$, and available thrust, are greatly mitigated.

The rest of this article is structured as it follows. In Section II the generalized UAV modeling is presented, while the generalized UAV estimation scheme is depicted in Section III. In Section IV the establishment of the proposed online parameter adaptive MPC is presented with Section V to present multiple simulation results that prove the efficiency of the suggested scheme are depicted, followed by a detailed discussion on the obtained results in Section VI, while the article concludes in Section VII. 


\section{GENERALIZED UAV MODELING}

In this Section, the overall modeling and the UAV frame, utilized in the simulations, will be established.

The modeling and estimation scheme have been initially established in [13]. However, to retain consistency, a reduced version and adapted to the specific aim of this article will be depicted in the sequel. Initially, the model for each motors' thrust is rewritten as a function of a normalized control signal $u_{M, i} \in[0,1]$ to be compatible with the todays Electronic Speed Controllers (ESCs), which accepts a 0 $100 \%$ reference signal.

$$
\begin{aligned}
\boldsymbol{F}_{i} & =A_{F, i} \Omega_{i, \max }^{2} u_{i}^{2} \hat{\boldsymbol{n}}_{i}, \\
\boldsymbol{\tau}_{i} & =-\operatorname{sgn}\left(\Omega_{i}\right) B_{F, i} \Omega_{i, \max }^{2} u_{i}^{2} \hat{\boldsymbol{n}}_{i}, \\
u_{i} & =\frac{1}{\tau_{i} s+1} u_{M, i},
\end{aligned}
$$

where $A_{F, i} \in \mathbb{R}_{+}$is the thrust constant, while $B_{F, i} \in \mathbb{R}_{+}$ is the torque constant, of the motor/propeller combination, $\Omega_{i, \max } \in \mathbb{R}_{+}$is the maximum rotational rate of the motor (which can be time dependent due to declining battery voltage), $\tau_{i} \in \mathbb{R}_{+}$is the time-constant of the motor/speed controller combination and $u_{M, i}$ is the commanded normalized rotor velocity, while finally $\hat{\boldsymbol{n}}_{i}$ is the motor directionality vector and $i$ corresponds to the $i^{\text {th }}$ motor. This simplified, but at the same time very accurate model has been initially presented in [13] to capture the majority of the effects in a real UAV system, however an addition is made, which was not included in [13], as the lower bound of the control signal is around 5\%, which comes from ESCs needing some motor rotation to operate. This induces a bound for the control signal that should be adhered to, if the motors should not stall in mid flight.

By considering the system from a normalized thrust perspective, it provides specific advantages in the later control design formulation, as it will presented in the sequel, where bounds on control signal can be easily considered, rather than on the motor's rotational rate, where $A_{F, i} \Omega_{i, \max }^{2}$ contains all the corresponding effects that affects the thrust. This includes the decline of the battery voltage, the wear on the motors' bearings or other mechanical changes, for cheap ESCs that do not have closed-loop control on the rotational rate. Moreover, it allows the estimation for compensation of the ground-effect, where the apparent thrust constant $A_{F, i}$ increases due to the air cushion being created below the UAV when flying close to the ground.

Following the parameterization that a nominal distance vector from the $\mathrm{CoG}$ to each motor is parameterized with the distance vector $\boldsymbol{l}_{i} \in \mathbb{R}^{3}$ and that the shift of CoG is parameterized with $\Delta \boldsymbol{l}^{B} \in \mathbb{R}^{3}$ as described in Figure 1, the following model from the motors to torque and thrust is produced by using equations (1-2) as:

$$
\left[\begin{array}{c}
\boldsymbol{F}_{\text {total }}^{B} \\
\boldsymbol{\tau}_{\text {total }}^{B}
\end{array}\right]=\left[\begin{array}{c}
\sum_{i=1}^{N} \boldsymbol{F}_{i} \\
\sum_{i=1}^{N}\left(\boldsymbol{l}_{i}+\Delta \boldsymbol{l}^{B}\right) \times \boldsymbol{F}_{i}+\boldsymbol{\tau}_{i}
\end{array}\right]
$$

where the sub-index $i$ corresponds to the $i^{t h}$ motor.

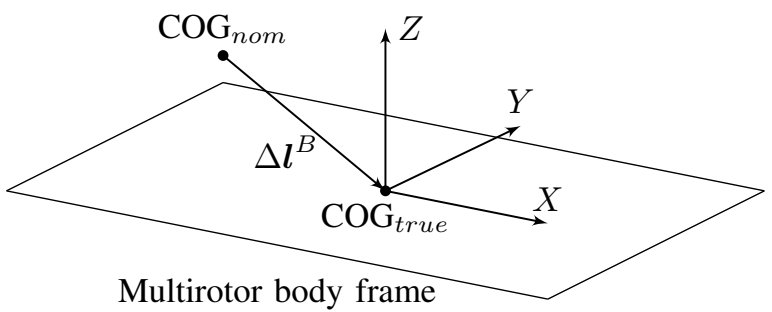

Fig. 1. A description of the offset COG from the body frame perspective.

Combining these modified models with the Newton-Euler equations, as described in [13] where the details of the derivation can be found, it results to a full model for the angular and linear acceleration of the UAV, with respect to the parameters and the control signal, as presented in (6), which is the basis for the presented estimation and control scheme.

However, this model has a drawback when it comes to the estimator and controller design, since the time-constant compensated control signal is squared. This introduces a non-linearity to an otherwise linear system that has to be taken under consideration. In this work it has been opted for moving the quadratic mapping to the input as:

$$
u_{i} \approx \frac{1}{\tau_{i} s+1} u_{i n, i}^{2},
$$

which preserves the static gain, while it has an effect on the time-constant, but has the strong merit that it makes the system linear. This will make the estimation and control scheme work in a $\boldsymbol{u}^{2}$ domain, while a square-root mapping is used before sending the control signals to the ESCs on the UAV as will be depicted in the overall system Figure 3.

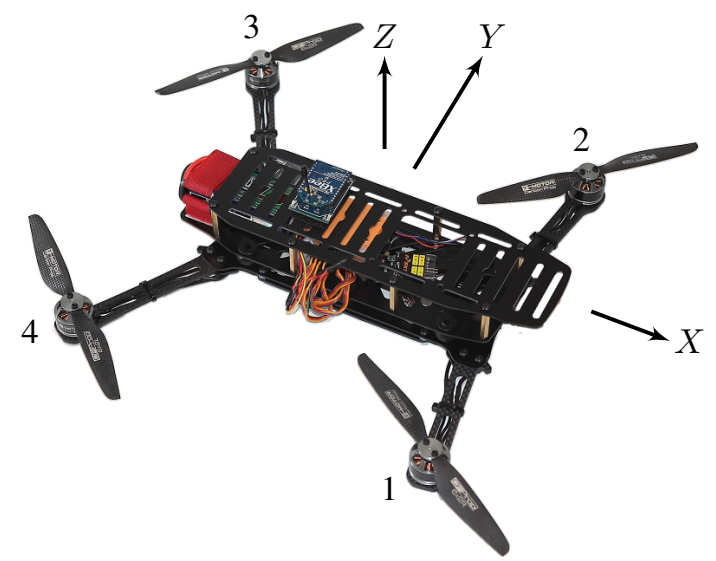

Fig. 2. The quadrotor whose model was used in the simulations, where motor 1,3 rotates counter clockwise, while motor 2, 4 rotates clockwise.

\section{A. UAV frame utilized}

The UAV frame utilized in this article is an X-type quadrotor, depicted in Figure 2, which is well used in the laboratory and has had full characterization of its model. The simple geometric shape of the UAV, where all motors are mounted flat, gives $\hat{\boldsymbol{n}}_{i}=\left[\begin{array}{lll}0 & 0 & 1\end{array}\right]^{\top} \forall i$. 


$$
\begin{aligned}
& {\left[\begin{array}{c}
\boldsymbol{a}^{B} \\
\dot{\boldsymbol{\omega}}^{B}
\end{array}\right]=\underbrace{\left[\begin{array}{ccc}
\cdots & \frac{A_{F, i} \Omega_{i, \max }^{2} \hat{\boldsymbol{n}}_{i}}{m} & \cdots \\
\cdots & \boldsymbol{I}_{c m}^{-1}\left[\left(\boldsymbol{l}_{i}+\Delta \boldsymbol{l}^{B}\right) \times A_{F, i} \Omega_{i, \max }^{2} \hat{\boldsymbol{n}}_{i}-\operatorname{sgn}\left(\Omega_{i}\right) B_{F, i} \Omega_{i, m a x}^{2} \hat{\boldsymbol{n}}_{i}\right] & \cdots
\end{array}\right]}_{\text {Allocation matrix } A} \underbrace{\left[\begin{array}{c}
\vdots \\
u_{i}^{2} \\
\vdots
\end{array}\right]}_{\boldsymbol{u}^{2}}+\left[\begin{array}{c}
\mathbf{0} \\
\boldsymbol{I}_{c m}^{-1}\left(\boldsymbol{\omega}^{B} \times \boldsymbol{I}_{c m} \boldsymbol{\omega}^{B}\right)
\end{array}\right]} \\
& u_{i}=\frac{1}{\tau_{i} s+1} u_{i n, i}
\end{aligned}
$$

\section{Parameter adaptive estimation}

Since the aim of this article is to compensate for the CoG movement and the corresponding changes in the thrust and mass, in the estimation scheme, not all the parameters of equation (6) are to be estimated. More specifically only the following parameters will be estimated:

$$
\boldsymbol{\beta}=\left[\begin{array}{c}
\Delta l_{x} \\
\Delta l_{y} \\
\frac{1}{N} \sum_{i=1}^{N} \frac{A_{F, i} \Omega_{i, \max }^{2}}{m}
\end{array}\right] \triangleq\left[\begin{array}{c}
\beta_{1} \\
\beta_{2} \\
\beta_{3}
\end{array}\right],
$$

where the sum comes form the fact that it is only possible to measure the sum of accelerations on the UAV, making only the mean possible to estimate. Moreover the angular acceleration and time-constant parameters defined as:

$$
\gamma=\frac{1}{N} \sum_{i=1}^{N}\left[\begin{array}{c}
\frac{A_{F, i} \Omega_{i, \max }^{2}}{I_{x x}} \\
\frac{A_{F, i} \Omega_{i, \max }^{2}}{I_{y y}} \\
\frac{B_{F, i}}{I_{z z}} \\
\tau_{i}
\end{array}\right] \triangleq\left[\begin{array}{c}
\gamma_{1} \\
\gamma_{2} \\
\gamma_{3} \\
\gamma_{4}
\end{array}\right]
$$

are assumed to be estimated beforehand from a system identification step, as discussed in [13], since the convergence of these parameters are highly dependent on that ample excitation exists.

For the estimation scheme, the dynamics of equation (6), combined with the simplification from equation (5) and the gyroscopic torques neglected, are integrated using a rectangle rule. Equation (3) is implemented as a discrete-time first order system, while the CoG and thrust parameters are modeled as integrated white noise, which results into the following prediction equations:

$$
\left[\begin{array}{c}
\boldsymbol{\omega}_{k} \\
\boldsymbol{u}_{k} \\
\boldsymbol{\beta}_{k}
\end{array}\right]=\left[\begin{array}{c}
\boldsymbol{\omega}_{k-1}+\Delta t \boldsymbol{A}_{\dot{\boldsymbol{\omega}}}\left(\beta_{1-2, k-1}, \gamma_{1-3}\right) \boldsymbol{u}_{k-1} \\
\frac{\gamma_{4}}{\Delta t+\gamma_{4}} \boldsymbol{A} \boldsymbol{u}_{k-1}+\frac{\Delta t}{\Delta t+\gamma_{4}} \boldsymbol{u}_{i n, k}
\end{array}\right]
$$

with the measurement vector defined as:

$$
\boldsymbol{z}_{k}=\left[\begin{array}{c}
\boldsymbol{\omega}_{k}^{B} \\
\beta_{3, k} \mathbf{1}_{1 \times 4} \boldsymbol{u}_{k}
\end{array}\right]
$$

where $\boldsymbol{A}_{\dot{\boldsymbol{\omega}}}$ is the angular acceleration acceleration part of the allocation matrix, while $\Delta t \in \mathbb{R}_{+}$is the sampling time in seconds. A standard Extended Kalman Filter (EKF) was chosen as the framework to estimate the states of the aforementioned system as it is bilinear, keeping the Jacobians simple. It should however be noted that the input $\boldsymbol{u}_{i n, k}$ is in the $\boldsymbol{u}^{2}$ domain, as depicted in Figure 3.

\section{Online Parameter Adaptive MPC Controller}

With the adaptive models provided by the estimation scheme and the aim to create a low-level controller for the angular rate and thrust tracking problem that is guaranteed by design to converge, without the need of integrators in the control loop, while adhering to control signal bounds, a time-varying linear MPC was selected.

With the estimation and control in the $\boldsymbol{u}^{2}$ domain, the model used in the MPC is a time-varying linear state-space of the following form:

$$
\begin{aligned}
& \boldsymbol{x}_{k}=\boldsymbol{A}_{k} \boldsymbol{x}_{k-1}+\boldsymbol{B} \boldsymbol{u}_{C, k}, \\
& y_{k}=\boldsymbol{C}_{k} \boldsymbol{x}_{k}+\boldsymbol{D} \boldsymbol{u}_{C, k}
\end{aligned}
$$

with the following time-varying model matrices defined as:

$$
\begin{aligned}
\boldsymbol{A}_{k} & =\left[\begin{array}{ll}
\boldsymbol{I}_{3 \times 3} & \Delta t \boldsymbol{A}_{\dot{\boldsymbol{\omega}}}\left(\beta_{1-2, k}, \gamma_{1-3}\right) \\
\mathbf{0}_{4 \times 4} & \frac{\gamma_{4}}{\Delta t+\gamma_{4}} \boldsymbol{I}_{4 \times 4}
\end{array}\right], \\
\boldsymbol{B} & =\left[\begin{array}{c}
\mathbf{0}_{4 \times 4} \\
\frac{\Delta t}{\Delta t+\gamma_{4}} \boldsymbol{I}_{4 \times 4}
\end{array}\right], \\
\boldsymbol{C}_{k} & =\left[\begin{array}{ll}
\mathbf{0}_{1 \times 3} & \beta_{3, k} \mathbf{1}_{1 \times 4}
\end{array}\right], \\
\boldsymbol{D} & =\left[\mathbf{0}_{1 \times 4}\right],
\end{aligned}
$$

which uses the parameters estimated in Section III, while $\boldsymbol{x}_{k}=\left[\begin{array}{ll}\hat{\boldsymbol{\omega}}_{k}^{\top} & \hat{\boldsymbol{u}}_{k}^{\top}\end{array}\right]^{\top}$ includes the angular rate and normalized rotational rate of the motors, and $y_{k}=a_{z, k}$ is the $\mathrm{z}$ acceleration. Finally the MPC is defined as the solution to the following convex optimization problem:

$$
\begin{aligned}
\underset{\left[\boldsymbol{x}_{k}, \boldsymbol{u}_{C, k}\right] \forall k}{\operatorname{minimize}} & \sum_{k=1}^{N}\left\|\boldsymbol{x}_{k}-\boldsymbol{x}_{k, d}\right\|_{\boldsymbol{Q}}^{2}+ \\
& \sum_{k=1}^{N}\left\|y_{k}-y_{k, d}\right\|_{P}^{2}+ \\
& \sum_{k=1}^{N}\left\|\boldsymbol{u}_{C, k}-\boldsymbol{u}_{C, k, d}\right\|_{\boldsymbol{R}}^{2}
\end{aligned}
$$

subject to equation (11),

$$
0.07^{2} \cdot \mathbf{1}_{4 \times 1} \leq \boldsymbol{u}_{C, k} \leq \mathbf{1}_{4 \times 1} \forall k
$$


where $N$ is the horizon, $P, \boldsymbol{Q}$, and $\boldsymbol{R}$ are positive definite weighting matrices, $\boldsymbol{x}_{k, d}$ is the desired states, while $y_{k, d}$ is the desired $z$-acceleration in the body frame of reference. In the defined cost, the lower bound of 0.07 comes from Section II, where the throttle needed for the motor to not stall was described, and a small extra throttle was added to keep the motor from stalling, resulting in a $7 \%$ as a bound for the minimum throttle. Finally $\boldsymbol{u}_{C, k, d}$ is the desired control signal that is, in contrary to the standard formulation, derived from the definition of "no net thrust nor angular rate" which can be described as:

$$
\boldsymbol{u}_{C, k, d}=\boldsymbol{M}\left[\begin{array}{llll}
0 & 0 & 0 & 1 \mathrm{~g}
\end{array}\right]^{\top},
$$

where $M$ is known as the mixing matrix and is defined, in the general case, as the pseudo-inverse of the allocation matrix in equation (6):

$$
\boldsymbol{M}=\boldsymbol{A}^{\top}\left(\boldsymbol{A} \boldsymbol{A}^{\top}\right)^{-1} .
$$

Overall this is a standard MPC with a time-varying model, that can be implemented easily and very efficiently with CVXGEN [14], a convex optimization solver generator providing highly optimized $\mathrm{C}$ code for later online use. A full overview of the proposed estimation and control combined architecture, while highlighting the $\boldsymbol{u}^{2}$ domain, is presented in Figure 3.

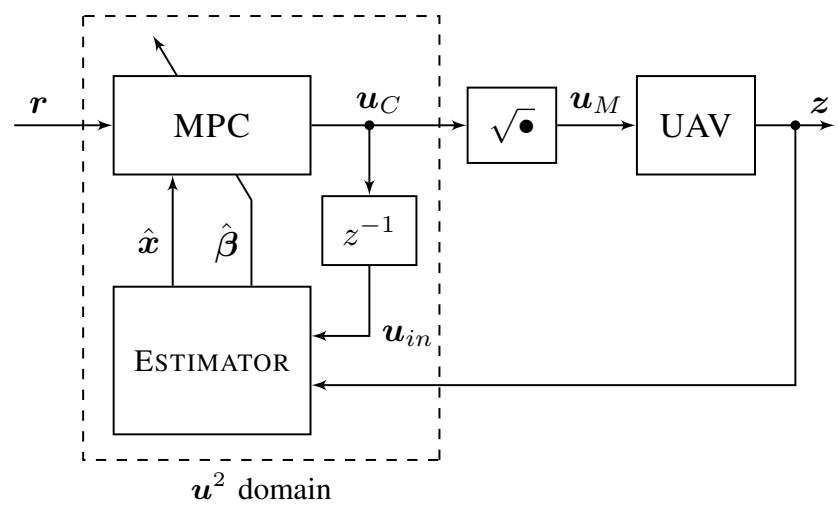

Fig. 3. A complete internal view of the estimation and control architecture, where the $\boldsymbol{u}^{2}$ domain is highlighted. The UAV block includes the dynamics of the UAV, the ESC model and the sensor models.

\section{Simulations}

\section{A. Tuning \& Parameters}

The tuning of the parameter estimation and control follows a straight forward method, which will be described there.

1) Model parameters: The parameters from (8) have been estimated previously through an off line identification scheme, as discussed in [13], while the lengths of the arms and mass are taken from the design of the quadrotor, and are presented in Table I, where $\mathrm{s}_{(\cdot)}$ and $\mathrm{c}_{(\cdot)}$ are the sine and cosine of the corresponding angle (.) in degrees. It should be noted that the mass is not used in the estimation nor the control scheme, it's only needed to calculate true values in the simulation. Moreover, all the parameters are spread $5 \%$ around their nominal values in the simulation, using
TABLE I

CONVERGED PARAMETER VALUES FOR THE UTILIZED QUADROTOR.

\begin{tabular}{cccc} 
Parameter & Unit & \multicolumn{2}{c}{ Parameter value } \\
\hline$\gamma_{1}$ & $\mathrm{rad} / \mathrm{s}^{2} / \mathrm{m}$ & 302 & \\
$\gamma_{2}$ & $\mathrm{rad} / \mathrm{s}^{2} / \mathrm{m}$ & 148 & \\
$\gamma_{3}$ & $\mathrm{rad} / \mathrm{s}^{2}$ & \multicolumn{2}{c}{11.7} \\
$\gamma_{4}$ & $\mathrm{~s}$ & 0.074 & \\
$\boldsymbol{l}_{1}$ & $\mathrm{~m}$ & $0.16 \cdot\left[\begin{array}{lll}\mathrm{s}_{-45} & \mathrm{c}_{-45} & 0\end{array}\right]^{\top}$ \\
$\boldsymbol{l}_{2}$ & $\mathrm{~m}$ & $0.16 \cdot\left[\begin{array}{lll}\mathrm{s}_{45} & \mathrm{c}_{45} & 0\end{array}\right]^{\top}$ \\
$\boldsymbol{l}_{3}$ & $\mathrm{~m}$ & $0.16 \cdot\left[\begin{array}{lll}\mathrm{s}_{135} & \mathrm{c}_{135} & 0\end{array}\right]^{\top}$ \\
$\boldsymbol{l}_{4}$ & $\mathrm{~m}$ & $0.16 \cdot\left[\begin{array}{lll}\mathrm{s}_{-135} & \mathrm{c}_{-135} & 0\end{array}\right]^{\top}$ \\
$m$ & $k g$ & 0.6 & \\
\hline$m$
\end{tabular}

a uniform distribution, to introduce the effects of model mismatch.

2) Estimation: The parameter estimation was tuned as follows:

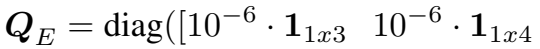

$$
\begin{aligned}
& \left.\left.10^{-8} \cdot \mathbf{1}_{1 \times 2} \quad 10^{-4}\right]\right) \\
& \boldsymbol{R}_{E}=\operatorname{diag}\left(\left[\begin{array}{ll}
0.0425^{2} \cdot \mathbf{1}_{1 x 3} & 0.0982^{2}
\end{array}\right]\right)
\end{aligned}
$$

where $\boldsymbol{Q}_{E}$ is the process covariance, and was tuned by trials until achieving a parameters' converged after approximately 0.5 seconds, it should be noted that below 0.5 seconds, excessive noise starts to enter the parameter estimation, while slower change of the parameters can also be useful in applications where fast changes to the $\mathrm{CoG}$ or mass are not expected. Meanwhile the $\boldsymbol{R}_{E}$ uses the measured sensor covariances and the starting state covariance was set to:

$$
\boldsymbol{P}_{0}=\operatorname{diag}\left(\left[\begin{array}{lll}
\mathbf{0}_{1 x 7} & 10^{-3} \cdot \mathbf{1}_{1 x 2} & 1
\end{array}\right]\right),
$$

based on typical values, usually calculated in corresponding experiments, where $\boldsymbol{Q}_{E}$ is of size $10 \times 10$, which comes from the angular rate states $\left(\omega_{3 \times 1}\right)$, the control signal states $\left(\boldsymbol{u}_{4 \times 1}\right)$, and the $\left(\boldsymbol{\beta}_{3 \times 1}\right)$, while $\boldsymbol{R}_{E}$ is of size $4 \times 4$ that comes from the angular rate measurements $\left(\boldsymbol{\omega}_{m, 3 \times 1}\right)$ and the accelerometer measurement in the $z$-axis $\left(a_{z, 1 \times 1}\right)$.

3) Control: While the MPC was tuned as follows:

$$
\begin{aligned}
& \boldsymbol{Q}=\operatorname{diag}\left(\left[\begin{array}{llll}
1 & 2 & 5 & 10^{-3} \cdot \mathbf{1}_{1 x 4}
\end{array}\right]\right) \\
& P=1 \\
& \boldsymbol{R}=\operatorname{diag}\left(\left[5 \cdot \mathbf{1}_{1 x 4}\right]\right) \\
& N=7
\end{aligned}
$$

the terminal weights were added as $\boldsymbol{Q}_{T}=10 \boldsymbol{Q}$ and $P_{T}=5 P$. The low weight on the normalized rotational rate state comes from the weights on the control signal, where the normalized rotational rates are simply low-pass filtered versions of the input, allowing the characteristics to be determined by the control signal weights alone. The normalized rotational rate weights can however not be 0 , as this would make the optimization problem non-convex. 


\section{B. Simulation results}

The simulations were carried out by having step changes, to the parameters, being made in three instances, with the aim to drive the system to its limits and force the controller to start hitting constraints. The three steps were carried out at $1 \mathrm{~s}, 1.5 \mathrm{~s}$ and $5 \mathrm{~s}$ into the simulation and were a CoG shift in $x$ direction, CoG shift in $y$ direction, and finally a signifiant mass change. The parameter changes are presented in Figure 6, which are also indicated in the corresponding time frame by the dashed vertical lines in Figures 4-6.
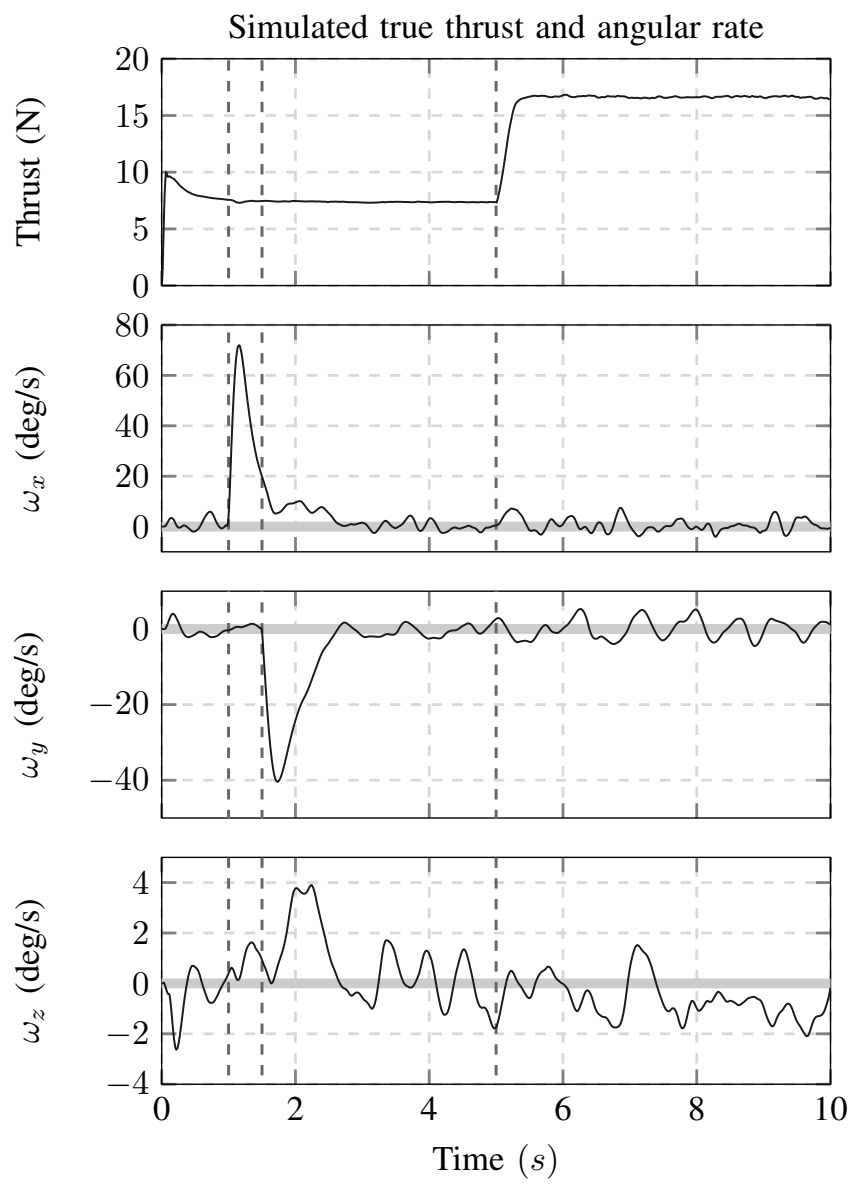

Fig. 4. In the sub-figures the thrust generated by the motors, and the angular around each axis is depicted. The solid black line is the simulated true value, while the thick gray line is reference.

As it can be observed in Figure 4, when the CoG shifts, corresponding errors in the angular rates are introduced, while these converge back to zero within a second due to the fast convergence of the $\mathrm{CoG}$ parameters. What should be noted at this point is in Figure 5, where the large movement of $\mathrm{CoG}$ forces the motor 2 to hit its lower constraint in order to keep the motor from stalling, while the other motors compensate. With motor 2 being so close to its lower bound, the overall situation introduces more noise in its control signal, as presented in the second sub-figure of Figure 5, which comes from the quadratic nature of thrust. In general, at small throttles, larger changes in the control signal are needed to produce the necessary changes in the thrust.
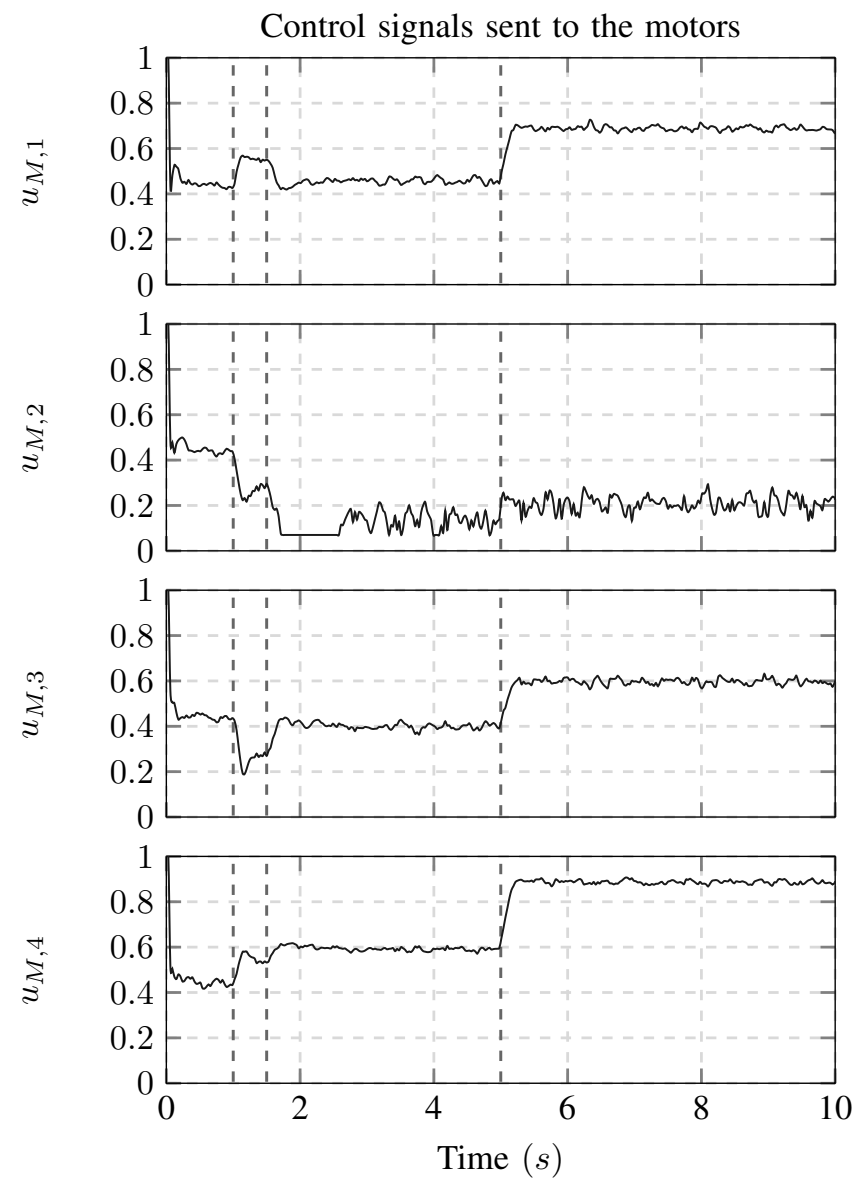

Fig. 5. In the sub-figures each of the motors' control signals are depicted.

Moreover, when the mass is increased, the thrust constant converges within 0.5 seconds, which corrects the thrust, and is clearly visible in Figure 4, while also is being visible as the control signals adapt to the new situation in Figure 5. The fast adaptation of thrust is of a paramount importance, especially when flying close to the ground and compensating for the ground effect, the air cushion created under the UAV, and gives an apparent increase of thrust.

It is worth to note the minor effect the changes of $\mathrm{CoG}$ have on the thrust, while the same is true for changes in mass and the angular rates. When the mass changes, only minor errors are introduced to the angular rates, which is caused by the small model mismatch introduced in Section V-A.1. Also by including the models for the reaction time of the motors it allows the controller to have a more fine grained control of the system, compared to the system neglecting these dynamics, which is quite common as it can be seen in, for example, [15].

Finally, as this estimation and control scheme only aims to control the angular rate and thrust, another layer on top of the suggested novel framework is needed if it's desired to control the attitude, or make a full control scheme for the translation. This is however not the aim of this article, while this work can be used as the low-level controller for other high-level schemes commonly found in the corresponding 

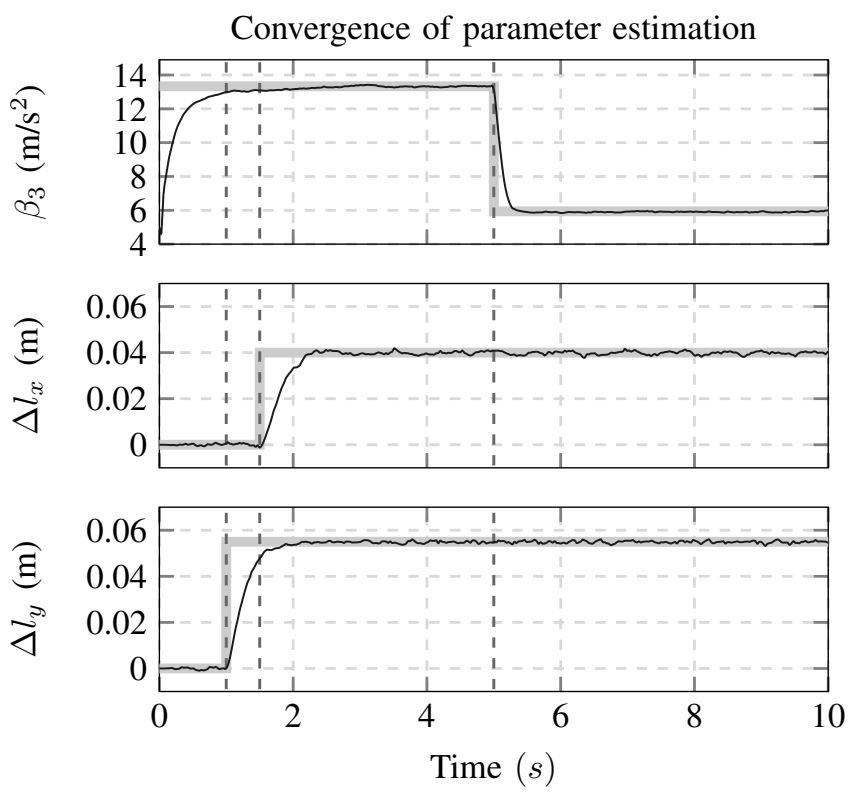

Fig. 6. In the first sub-figure the thrust constant estimation results are depicted, while in the final two sub-figures the $\mathrm{CoG}$ estimation is presented. The solid black line is the simulated true value, while the thick gray line is the calculated true value.

literature.

\section{DISCUSSION}

What has not been discussed in the article is the effect of parameter bounds, where the thrust constant must be positive and the CoG parameters must be inside the motor centers. These bounds can easily be added, however these are implementation specific issues that come with the selection of the estimator scheme where, for example, an optimization based estimation has the ability to include bounds directly.

It should be also mentioned that a classical PID controller, combined with a mixing matrix, was not selected due to the fact that the PID performs badly when the mixing matrix changes, which is a fact that introduces cross couplings between axises from the moving CoG. In the case that a thrust is requested that saturates the system, then the controllability of the angular rates is lost, as there is no control authority left. To compensate for this this problem the control engineers commonly add gain scheduling or back off strategies, as for example it can be seen in [16], where a control back off scheme has been introduced, called "Air Mode", which backs off the throttle if there is a torque requested that otherwise would be saturated by the thrust and thus providing full priority to torques. From another point of view, in an MPC scheme, as the one presented, these drawbacks do not exist and a back off strategy is not necessary, as the weight matrices of the cost function allows for any selection of priorities between the thrust and torque, and furthermore the weights can be changed based on the situation specifics and thus allowing for an overall fine tuned control in specialized cases without further additions to the controller itself.

\section{CONCLUSIONS}

In this article an adaptive estimation and control scheme has been developed and tested in simulations pushing the UAV system to its limits. The system has the significant merit of being robust and fast to track corresponding changes in the CoG and thrust constant of the UAV. This is achieved by utilizing an online estimation of a generalized model of a multirotor UAVs, where the parameter estimation and the adaptive model is used by an MPC to reject disturbances and track references, while at the same time retaining the control signal bounds not to have the motors stall. The linear requirements of the MPC are adhered to by having the estimation and control working in a $\boldsymbol{u}^{2}$ domain, where the dynamics and models are linear.

\section{REFERENCES}

[1] K. Alexis, G. Nikolakopoulos, A. Tzes, and L. Dritsas, "Coordination of helicopter UAVs for aerial Forest-Fire surveillance," in Applications of Intelligent Control to Engineering Systems. Springer Netherlands, June 2009, pp. 169-193.

[2] S. S. Mansouri, C. Kanellakis, D. Wuthier, E. Fresk, and G. Nikolakopoulos, "Cooperative aerial coverage path planning for visual inspection of complex infrastructures," arXiv preprint arXiv:1611.05196, 2016.

[3] C. Kanellakis and G. Nikolakopoulos, "Survey on Computer Vision for UAVs: Current Developments and Trends," Journal of Intelligent \& Robotic Systems, pp. 1-28, 2017.

[4] P. E. Pounds, D. R. Bersak, and A. M. Dollar, "Grasping from the air: Hovering capture and load stability," in Robotics and Automation (ICRA), 2011 IEEE International Conference on. IEEE, 2011, pp. 2491-2498.

[5] Q. Lindsey, D. Mellinger, and V. Kumar, "Construction with quadrotor teams," Autonomous Robots, vol. 33, no. 3, pp. 323-336, 2012.

[6] S. Shimahara, R. Ladig, L. Suphachart, S. Hirai, and K. Shimonomura, "Aerial manipulation for the workspace above the airframe," in Intelligent Robots and Systems (IROS), 2015 IEEE/RSJ International Conference on, Sept 2015, pp. 1453-1458.

[7] N. Michael, J. Fink, and V. Kumar, "Cooperative manipulation and transportation with aerial robots," Autonomous Robots, vol. 30, no. 1, pp. 73-86, 2011.

[8] E. Fresk and G. Nikolakopoulos, "Full Quaternion Based Attitude Control for a Quadrotor," European Control Conference, 2013.

[9] K. Alexis and G. Nikolakopoulos and A. Tzes, "Model predictive quadrotor control: attitude, altitude and position experimental studies," IET Control Theory Applications, vol. 6, no. 12, pp. 1812-1827, Aug 2012.

[10] P. Pounds, R. Mahony, and P. Corke, "System identification and control of an aerobot drive system," in Information, Decision and Control, 2007. IDC'07. IEEE, 2007, pp. 154-159.

[11] N. Abas, A. Legowo, Z. Ibrahim, N. Rahim, and A. M. Kassim, "Modeling and system identification using extended kalman filter for a quadrotor system," in Applied Mechanics and Materials, vol. 313. Trans Tech Publ, 2013, pp. 976-981.

[12] D. Mellinger, Q. Lindsey, M. Shomin, and V. Kumar, "Design, modeling, estimation and control for aerial grasping and manipulation," in 2011 IEEE/RSJ International Conference on Intelligent Robots and Systems, Sept 2011, pp. 2668-2673.

[13] E. Fresk, D. Wuthier, and G. Nikolakopoulos, "Generalized center of gravity compensation for multirotors with application to aerial manipulation," in 2017 IEEE/RSJ International Conference on Intelligent Robots and Systems (IROS). IEEE, 2017, pp. 16-23.

[14] J. Mattingley and S. Boyd, "Cvxgen: A code generator for embedded convex optimization," Optimization and Engineering, vol. 13, no. 1, pp. 1-27, 2012.

[15] D. Mellinger and V. Kumar, "Minimum snap trajectory generation and control for quadrotors," in Robotics and Automation (ICRA), 2011 IEEE International Conference on. IEEE, 2011, pp. 2520-2525.

[16] Cleanflight Open Source Racing UAV controller, http://www.cleanflight.com/. 